\title{
Development of fully 3D image reconstruction techniques for pinhole SPECT imaging
}

\author{
ZHANG XueZhu ${ }^{1,2 *} \&$ QI YuJin ${ }^{1 *}$ \\ ${ }^{1}$ Shanghai Institute of Applied Physics, Chinese Academy of Sciences, Shanghai 201800, China; \\ ${ }^{2}$ Graduate University of Chinese Academy of Sciences, Beijing 100049, China
}

Received February 5, 2010; accepted March 7, 2010

\begin{abstract}
The purpose of this study was to develop fully 3D image reconstruction techniques for pinhole SPECT imaging with our Micro-SPECT system. Our studies involve in the derivation of projection operators, analysis of the sampling characteristics of pinhole SPECT imaging in Radon space, development of effective geometric calibration method for system misalignment, and 3D image reconstruction development and implementation with quantitative degrading compensation for pinhole SPECT with both circular and helical scan. The performances of pinhole SPECT imaging were evaluated using computer simulations and experiments with the Ultra-Micro Hot-Spot phantom, Ultra-Micro Defrise phantom and small-animal imaging. The results from the computer simulations and phantom imaging experiments indicate that the statistically-based iterative algorithms with quantitative compensation provide overall image quality improvement, and the system resolution is significantly recovered for quantitative imaging. The helical pinhole SPECT improves the axial field-of-view (FOV) as compared with the standard pinhole SPECT with circular-orbit scan. The mouse bone imaging experiment shows that the helical pinhole SPECT imaging also provides decent high-resolution whole-body small animal imaging. In conclusion, we have successfully developed a set of valid fully 3D image reconstruction techniques for single-pinhole SPECT imaging. These techniques can be easily extended to multi-pinhole SPECT imaging.
\end{abstract}

pinhole SPECT, fully 3D image reconstruction, sufficient sampling, quantitative compensation, geometric calibration, computer simulation

Citation: Zhang X Z, Qi Y J. Development of fully 3D image reconstruction techniques for pinhole SPECT imaging. Chinese Sci Bull, 2011, 56: 340-348, doi: $10.1007 / \mathrm{s} 11434-010-3200-\mathrm{x}$

In recent years single photon emission computed tomography (SPECT) for in vivo small animal imaging has gained great significance in the pre-clinical molecular imaging research for medicine and biology studies [1-4]. To achieve high spatial resolution and improved detection efficiency, pinhole collimator instead of the conventional parallel-hole collimator has been widely utilized for the development of dedicated small-animal SPECT systems (also called MicroSPECT) [5-8].

Although pinhole imaging can deliver high spatial resolution and improved detection efficiency when imaging small object close to the pinhole aperture, this will be at the

*Corresponding authors (email: xuezhu.zhang@ieee.org; qiyujin@sinap.ac.cn) cost of the size of the field-of-view (FOV) of the imaging object. As compared with the clinical SPECT with parallel-hole collimation, pinhole SPECT has a different imaging geometry with magnification and limited FOV which results in insufficient sampling $[9,10]$ in the tomographic projection data acquisition. So there is a great challenge in the development of fully $3 \mathrm{D}$ image reconstruction techniques for pinhole SPECT imaging.

In this study, we aimed to develop fully 3D image reconstruction techniques for quantitative pinhole SPECT imaging with our Micro-SPECT system. The projection operators of pinhole SPECT imaging was derived with coordinate transformation of geometric parameters. Then the sampling characteristics and sufficient condition of pinhole SPECT 
imaging were investigated in Radon space and compared in different acquisition modes with circular-orbit and helical-orbit scans. Fully 3D image reconstruction for quantitative pinhole SPECT was developed using statistically-based iterative method with effective degrading compensation. Geometric calibration about system misalignment also was studied and implemented for reconstruction by accurate coordinate parameters. The performances of our developed image reconstruction techniques of pinhole SPECT were evaluated using computer simulations, phantom experiments and small animal imaging experiment by our MicroSPECT system as compared with the pinhole SPECT with analytical reconstruction algorithm and circular scan mode.

\section{Methods and materials}

\subsection{Projection operators of pinhole SPECT}

The mathematical description about image formation and reconstruction of pinhole SPECT refers to the coordinate transformation in specific imaging geometry. Given that the system is stationary, especially the axis of rotation (AOR) is not wobbled and the imaged object rotates across accurate equiangular interval. For convenient derivation, we define the projection operators of pinhole SPECT in Cartesian coordinate systems. As shown in Figure 1, Oxyz and $O^{\prime \prime \prime} x^{\prime \prime \prime} y^{\prime \prime \prime} z^{\prime \prime \prime}$ denote the Detector Imaging Reference System (DIRS) and the Object Reference System (ORS) respectively. We consider that the origin $\mathrm{O}$ of the DIRS is at the center of the practical detector plane. We assume that $O^{\prime \prime \prime} y^{\prime \prime \prime / / ~} z O y$ and the origin $O^{\prime \prime \prime}$ of the ORS (also the AOR) is in the coordinate plane $x O y$ of the DIRS. We define 7 geometric parameters for single-pinhole SPECT as listed in Table 1: the tilted angle $\alpha$ from $O^{\prime \prime \prime} x$ "' to $O x$, the screwed angle $\beta$ from $O^{\prime \prime \prime} y^{\prime \prime \prime}$ to $O y$, the coordinate $\left(x_{c}, y_{c}, z_{c}\right)$ of the origin $O^{\prime \prime \prime}$ of the ORS in the DIRS (where $z_{c}=0$ ), and the coordinate $\left(x_{p}, y_{p}, z_{p}\right)$ of the focal center of pinhole collimator (PH) in the DIRS, in which $x_{p}<x_{c}$. In addition, the symbol $\theta$ denotes the angle of the imaging object rotated relative to the start angle $\theta_{0}$. The object rotation could be described as eq.(1), where ( $x^{\prime \prime \prime}, y^{\prime \prime \prime}$, $\left.z^{\prime \prime \prime}\right)$ and $\left(x_{\theta}^{\prime \prime \prime}, y_{\theta}^{\prime \prime \prime}, z_{\theta}^{\prime \prime \prime}\right)$ denote the coordinates of a point object at its initial position and after the angle $\theta$ of rotation in the ORS respectively, and the operator $R_{\theta}$ is the coordinate transformation of object rotation in the ORS. Hereafter, $\alpha, \beta$ and $\theta$ are all assumed as clock-wise angles.

Table 1 Defined geometric parameters of projection operators for pinhole SPECT imaging

\begin{tabular}{cc}
\hline Symbol & Description \\
\hline$\left(x_{c}, y_{c}\right)$ & origin coordinate of AOR in the DIRS $\left[z_{c}=0\right]$ \\
$\left(x_{p}, y_{p}, z_{p}\right)$ & coordinate of PH in the DIRS \\
$\alpha$ & tilted angle from $O^{\prime \prime \prime} x^{\prime \prime \prime}$ to $O x$ \\
$\beta$ & screwed angle from $O^{\prime \prime \prime} y^{\prime \prime \prime}$ to $O y$ \\
$\theta$ & angle of rotation [known] \\
\hline
\end{tabular}

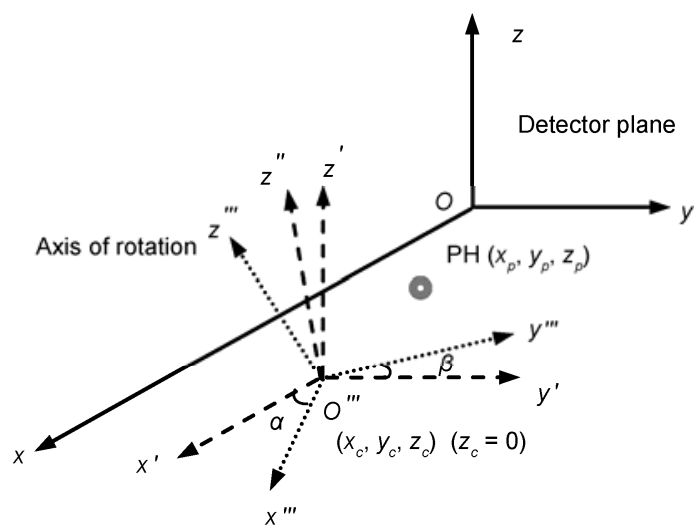

Figure 1 The schematic of the coordinate transformation from the object reference system (ORS) to the detector imaging reference system (DIRS) of pinhole SPECT imaging.

$$
R_{\theta}=\left[\begin{array}{cccc}
\cos \theta & \sin \theta & 0 & 0 \\
-\sin \theta & \cos \theta & 0 & 0 \\
0 & 0 & 1 & 0 \\
0 & 0 & 0 & 1
\end{array}\right], \quad\left[\begin{array}{c}
x_{\theta}^{\prime \prime \prime} \\
y_{\theta}^{\prime \prime \prime} \\
z_{\theta}^{\prime \prime \prime} \\
1
\end{array}\right]=R_{\theta} \times\left[\begin{array}{c}
x^{\prime \prime \prime} \\
y^{\prime \prime \prime} \\
z^{\prime \prime \prime} \\
1
\end{array}\right] .
$$

The coordinate transformation from the ORS to the DIRS relates to two angle rotations $(\alpha$ and $\beta$ ) and one translation $\left(x_{c,} y_{c}, z_{c}\right)$, derived and expressed in eqs. (2)-(5). $\left(x_{\theta}^{\prime \prime \prime}, y_{\theta}^{\prime \prime \prime}, z_{\theta}^{\prime \prime \prime}\right)$ and $\left(x_{\theta}, y_{\theta}, z_{\theta}\right)$ are the coordinates of the point in the ORS and in the DIRS respectively by the multiplication of the operators $R_{\alpha}, R_{\beta}$ and $T$. As discussed above, when the origin of three ORS is in the coordinate plane $x O y$ of the DIRS, we have $z_{c}=0$.

$$
\begin{gathered}
R_{\alpha}=\left[\begin{array}{cccc}
\cos \alpha & 0 & \sin \alpha & 0 \\
0 & 1 & 0 & 0 \\
-\sin \alpha & 0 & \cos \alpha & 0 \\
0 & 0 & 0 & 1
\end{array}\right],\left[\begin{array}{c}
x_{\theta}^{\prime \prime} \\
y_{\theta}^{\prime \prime} \\
z_{\theta}^{\prime \prime} \\
1
\end{array}\right]=R_{\alpha} \times\left[\begin{array}{c}
x_{\theta}^{\prime \prime \prime} \\
y_{\theta}^{\prime \prime \prime} \\
z_{\theta}^{\prime \prime \prime} \\
1
\end{array}\right], \\
R_{\beta}=\left[\begin{array}{cccc}
1 & 0 & 0 & 0 \\
0 & \cos \beta & -\sin \beta & 0 \\
0 & \sin \beta & \cos \beta & 0 \\
0 & 0 & 0 & 1
\end{array}\right], \quad\left[\begin{array}{c}
x_{\theta}^{\prime} \\
y_{\theta}^{\prime} \\
z_{\theta}^{\prime} \\
1
\end{array}\right]=R_{\beta} \times\left[\begin{array}{c}
x_{\theta}^{\prime \prime} \\
y_{\theta}^{\prime \prime} \\
z_{\theta}^{\prime \prime} \\
1
\end{array}\right], \\
T=\left[\begin{array}{llll}
1 & 0 & 0 & x_{c} \\
0 & 1 & 0 & y_{c} \\
0 & 0 & 1 & z_{c} \\
0 & 0 & 0 & 1
\end{array}\right], \quad\left[\begin{array}{c}
x_{\theta} \\
y_{\theta} \\
z_{\theta} \\
1
\end{array}\right]=T \times\left[\begin{array}{c}
x_{\theta}^{\prime} \\
y_{\theta}^{\prime} \\
z_{\theta}^{\prime} \\
1
\end{array}\right], \\
{\left[\begin{array}{ll}
x_{\theta} y_{\theta} z_{\theta} & 1
\end{array}\right]^{\mathrm{T}}=T \times R_{\beta} \times R_{\alpha} \times R_{\theta} \times\left[x^{\prime \prime \prime} y^{\prime \prime \prime} z^{\prime \prime \prime} 1\right]^{\mathrm{T}} .}
\end{gathered}
$$

The projection operators of pinhole imaging are derived and presented in eq. (6). $\left(x_{d}, y_{d}, z_{d}\right)$ denotes the projection coordinate of the point object projected through the pinhole collimator to the detector plane in the DIRS where $x_{d}=0$. 


$$
\begin{aligned}
& y_{d}=y+\left(\frac{x_{d}-x}{x-x_{p}}\right)\left(y-y_{p}\right), \\
& z_{d}=z+\left(\frac{x_{d}-x}{x-x_{p}}\right)\left(z-z_{p}\right) .
\end{aligned}
$$

In the following discussion, we will implement the computer simulation, image reconstruction, and geometric calibration of pinhole SPECT imaging based on the above reference framework of the coordinate transformation.

\subsection{Sampling characteristics of pinhole SPECT}

The pinhole SPECT has almost the same imaging geometry as the cone-beam CT. There exists a sufficient sampling issue of projections for fully $3 \mathrm{D}$ image reconstruction. In the 1980s, Tuy et al. respectively derived the necessary and sufficient mathematical condition for analytical cone-beam tomographic reconstruction [11-13]. The sufficient condition states that if the normal plane of every point in the support intersects with at least one point of the cone-beam source trajectory, the fully $3 \mathrm{D}$ reconstruction could be achieved in the whole object. This condition is presented as the mathematical eq. (7), where $\Phi(\lambda)$ represents the function of the source trajectory and $\boldsymbol{n}$ is the normal vector of the point $x$ in the support to be reconstructed.

$$
\langle x, \boldsymbol{n}\rangle=\langle\Phi(\lambda), \boldsymbol{n}\rangle .
$$

The sufficient condition of pinhole SPECT imaging has been analysed by some studies, whereas the sampling characteristics can be more intuitively illustrated in the Radon Space which denotes the range of 3D Radon transform. Every value in Radon space represents unique plane integral of 3D Radon transform passing through a given point in the object region, and the value is defined as Radon Value. To achieve fully $3 \mathrm{D}$ reconstruction, sufficient Radon values in the object should be obtained. Figure 2 shows the schematics about the 3D Radon Space of single-pinhole SPECT imaging, which is the aerial perspective (a), trans-axial central image (b), and axial central images of 3D Radon space with circular scan (c) and helical scan (d) respectively. In the helical scan mode, more Radon values are sampled along the axis of rotation, resulting in more sufficient sampling space than the circular scan. In the meanwhile, the trans-axial sampling of 3D Radon Space is relatively uniform as well. Therefore, the helical scan can be an alternative method to the routine circular scan and provide extended axial FOV for imaging the long object for fully 3D image reconstruction.

\subsection{Image reconstruction methods}

The image formation of SPECT could be approximately described by the mathematical methods as the continuous
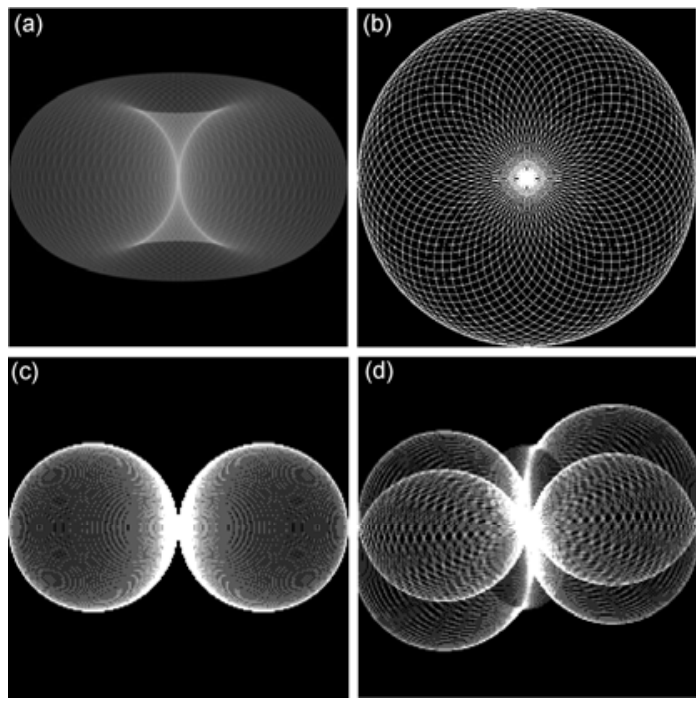

Figure 2 3D Radon space of single-pinhole SPECT with circular scan and helical scan. (a) Aerial perspective rending; (b) the trans-axial central image; (c) the axial central image of circular scan; (d) the axial central image of helical scan.

integral model or the discrete projection equations about photon emission of the concentrated radioactive tracer in vivo from the object to the detector [14]. These mathematical methods refer to the study of inverse problems about image reconstruction from projections [15-17]. In computed tomography, the analytical image reconstruction methods have been studied extensively and developed greatly, such as the non-exact filtered back-projection (FBP) methods are widely used in X-ray CT imaging. However, in emission computed tomography, statistically-based iterative image reconstruction methods can provide improved image quality by more accurate physical and statistical modeling of gamma photon production and detection processes as compared with the analytic image reconstruction methods. Among the statistical iterative reconstruction algorithms, the Maximum Likelihood Expectation Maximization (ML-EM) algorithm is a well developed one and currently widely used in the image reconstruction of emission tomography [18-20]. This numerical iterative method is based on Poisson statistical process of photon imaging and derived from maximizing the expectation of the likelihood function about the probability of mean photon counts. However, its computational convergence is very slow. In order to overcome this problem, Ordered Subsets EM (OS-EM) method has been proposed to accelerate the convergence of ML-EM, where the projection data need to be divided and ordered into appropriate subsets in the reconstruction procedure [21]. The general algorithm can be described as the following iterative eq. (8).

$$
\bar{f}_{\mathrm{i}}^{(k+1)}=\frac{\bar{f}_{i}^{(k)}}{\sum_{j \in S_{n}} C_{i j}} \sum_{j \in S_{n}} \frac{g_{j}}{\sum_{i=1}^{m} C_{i j} \bar{f}_{i}^{(k)}} C_{i j},
$$


In eq. (8), $C_{i j}$ represents the probability of one photon emitted from the $i$ th image voxel and detected by the $j$ th detector bin. $\bar{f}_{i}^{(k+1)}$ is the $(k+1)$ th iterated estimated activity values of the $i$ th image voxel. $S_{n}$ denotes the $n$th $S$ ubset. This iteration procedure relates to the forward projection operation of current estimation, the back-projection operation of the estimated ones by the ratios of the measured counts $g_{j}$, then the operations of regularization and modification about pre-iterated estimated value. The estimated values of the object image are updated from the sub-iteration of each subset, and a single iteration will be finished when all subsets have been processed.

Because the small size of the imaging object such as mouse or rat, the magnitude of photon attenuation and scatter effects are much less significant than that in the human body. The collimator-detector response is the major factor of imaging degradation for the small-animal imaging, therefore the attenuation and scatter effects in the object and scatter in the collimator are not considered in our implemented reconstruction algorithm. Based on the projection operation of pinhole SPECT imaging, we developed several image reconstruction algorithms, including the Feldkamp algorithm (an analytical cone-beam FBP-like image reconstruction method) and the dedicated iterative voxel-driven 3D pinhole OS-EM algorithm for fully 3D image reconstruction with compensation for both circular and helical scan modes. In the implementation, we numerically calculate the approximate system response matrix, used as the keel-edge pinhole collimator-detector response compensation with geometric and penetration effects (GPRC) [22]. Figure 3 shows the geometric response (a) which is the overlap of photon emitted through the front aperture and the back aperture to the detector plane, and the penetrative response (b) which is the attenuation of photon passing through the edge of the pinhole collimator.

\subsection{Geometric parameters estimation}

In order to achieve accurate quantitative image reconstruction, the refined geometric calibration of system misalignment is very important for high-resolution pinhole SPECT imaging. We have developed an effective optimization me-
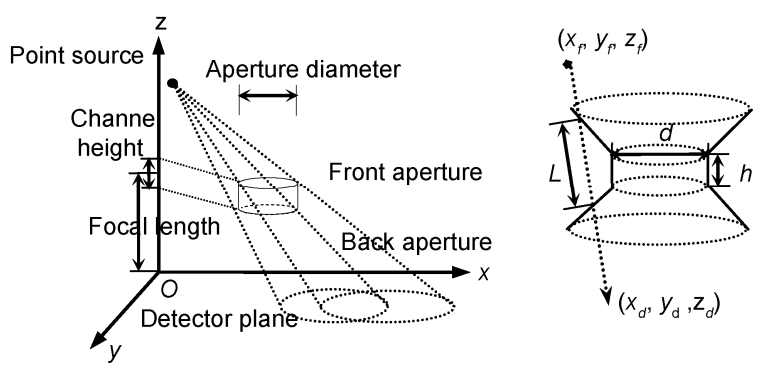

Figure 3 The schematic of the geometric point response (a) and the penetrative point response (b) of the keel-edge pinhole collimator. thod to estimate the complete seven parameters $\left(\alpha, \beta, x_{c}, y_{c}\right.$, $\left.x_{p}, y_{p}, z_{p}\right)$ in the geometric calibration for $3 \mathrm{D}$ image reconstruction of single-pinhole SPECT [23]. To estimate these geometric parameters, at least three point objects (or sources) phantom with a priori knowledge of relative point position information such as the known distance of each point is necessary and sufficient to constrain the optimization procedure to converge to a unique solution with a general scheme [24-26]. The cost function is structured as the least-squares about the residual error of measured and estimated projections of point object [the first term in eq.(9)], in which $m$ is the projection view $(m=1,2, \ldots, M), n$ is the $n$th point object $(n=1,2,3),\left(y^{i m g}, z^{i m g}\right)$ and $\left(y^{e s t}, z^{\text {est }}\right)$ represents the experimental measured values and estimated values of the projection coordinate of the point object respectively. If the prior information about the known distance of each point is not precise enough, then we should use this constraint condition as a second term and add it into the cost function of eq.(31) in [24]. The second term could be constructed as a weight coefficient $W$ multiplied by the sum of least-squares about the error between the known and estimated distance of each point. This cost function is described in eq. (9).

$$
\begin{aligned}
\min F= & \sum_{m} \sum_{n}\left[\left(y_{m n}^{i m g}-y_{m n}^{e s t}\right)^{2}+\left(z_{m n}^{i m g}-z_{m n}^{e s t}\right)^{2}\right] \\
& +W \cdot\left[\left(d_{12}-d_{12}^{\prime}\right)^{2}+\left(d_{13}-d_{13}^{\prime}\right)^{2}+\left(d_{23}-d_{23}^{\prime}\right)^{2}\right] .
\end{aligned}
$$

Accordingly we designed a series of general-purpose point-object-holder phantoms for geometric calibration procedure. The point sources are usually made by micro-spherical resins soaked with condensed radioactive solutions, and successive projections acquired over 360 degrees after the routine pinhole SPECT imaging experiments. Figure 4 shows a sample projection (a) and the superimposed projections (b) of 3 condensed point sources phantom with the preset distance of each point by our Micro-SPECT system. In this study, we choose the Powell algorithm for the implementation of the nonlinear iterative minimization [27], and the initial estimates should be chosen at the stable zone so as to constrain the optimization problem to converge to a global minimum.
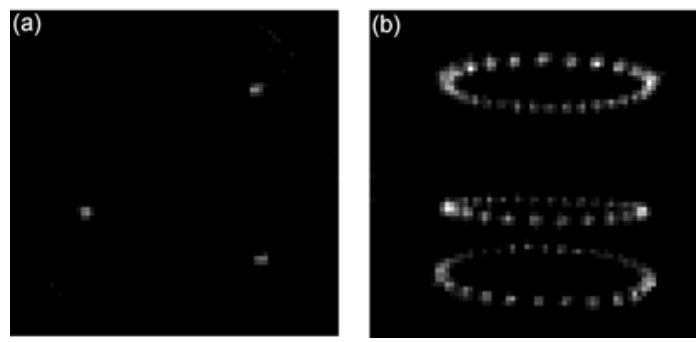

Figure 4 The projection of condensed point sources phantom with known distance of each point. (a) A sample projection of 3 point sources imaged by our Micro-SPECT system; (b) the superimposed projections of 30 views over 360 degree. 


\subsection{Micro-SPECT system}

The Micro-SPECT system developed in our lab consists of a stationary mini-gamma camera with pinhole collimator and vertical object rotation mechanism [28-30], as shown in Figure 5. The mini gamma camera is based on a $5 \mathrm{~mm}$ thick $80 \times 80$ pixellated $\mathrm{NaI}(\mathrm{Tl})$ scintillation crystal array with 1.2 $\mathrm{mm}$ pixel size and $0.2 \mathrm{~mm}$ gap (1.4 mm pixel pitch), coupled to a 5" diameter Hamamatsu R3292 position sensitive photomultiplier tube (PSPMT). The available dimensions of the projection data are taken as $70 \times 70$ detector bins. The pinhole collimator was optimized for $140 \mathrm{keV}$ gamma-rays with keel-edge aperture and an acceptance angle of $\sim 60^{\circ}$. The pinhole apertures are made of tungsten alloy filled with $0.5 \mathrm{~mm}$ channel height and interchangeable diameters of 0.7 , $1.0,1.5$ and $2.0 \mathrm{~mm}$. The focal length is $100 \mathrm{~mm}$ which results in a magnification of 2-3 for the routine experiments. The rotary stage was mounted on a linear translation stage to provide vertical translation during the object rotation for the helical SPECT scan. The helical pinhole SPECT imaging can be obtained in such a way where the object is translated longitudinally during the circular rotation to yield a helical orbit.

\section{Experiments and results}

To validate the fully $3 \mathrm{D}$ image reconstruction for pinhole SPECT, we made a series of performance comparison studies using computer simulations, phantom imaging experiments and small animal imaging experiments with both circular and helical scans. We implemented the Feldkamp algorithm, the dedicated 3D OS-EM algorithm with keel-edge pinhole collimator-detector response compensation, and also with circular and helical scans. These algorithms will be utilized in these experiments and compared in the following discussion.

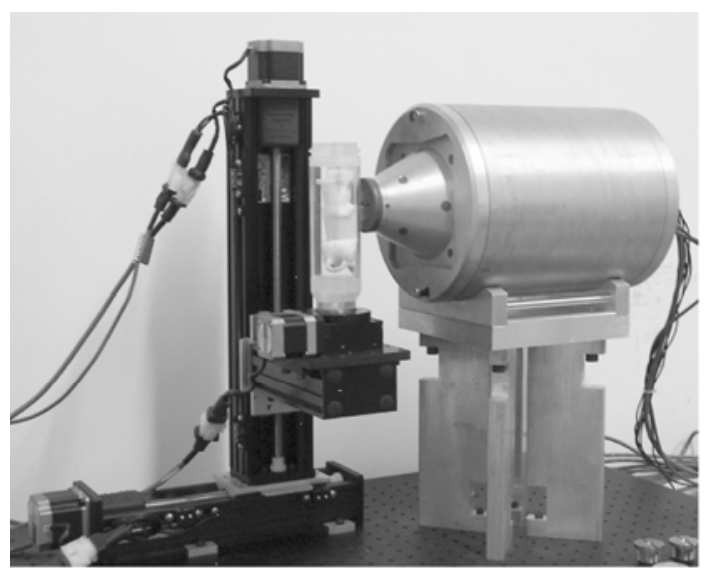

Figure 5 A photograph of the Micro-SPECT imaging system developed in our lab at SINAP.

\subsection{Computer simulations}

Computer simulations are very important for the implementation and evaluation of various image reconstruction methods, especially before developing practical imaging systems. To validate the performance of the reconstruction algorithms developed for pinhole SPECT imaging, two kinds of computerized phantoms were performed to assess the trans-axial reconstructed resolution and the axial reconstructed resolution, with circular scan and helical scan respectively. The imaging parameters of the simulation were based on the configuration of our Micro-SPECT system which was described in section 1.5.

A mathematical 3D Jaszczak phantom was first simulated to evaluate the performance of Feldkamp algorithm and the 3D pinhole OS-EM algorithm with the degrading compensation method which we introduced in section 1.3 and [22]. The phantom is based on the Ultra-Micro Hot Spot Phantom (Data Spectrum, Hillsborough, NC, USA), which consists of 6 sets of different diameter of hot spot channels: $2.4,2.0,1.7,1.35,1.0$ and $0.75 \mathrm{~mm}$ which simulates the filling of radioactive solutions for hot spot emission imaging. The spacing between adjacent spots is twice of the diameter in each set of channels. The diameter of the cylindrical insert is $27 \mathrm{~mm}$, the inside diameter of the phantom is $28 \mathrm{~mm}$, and the inside height is $9.9 \mathrm{~mm}$. We also simulated the bottom hot layer of which the thickness is 2 $\mathrm{mm}$. Figure 6(a) shows the trans-axial image of the computerized phantom, and (b) shows its 3D perspective rendering. The noise-free projections were generated with 1.0 $\mathrm{mm}$ keel-edge pinhole collimator, of which a sample projection is shown in Figure 6(c). Then the data were blurred further with Poisson statistical distribution and noise contaminated, as shown in Figure 6(d). No scatter and attenuation effects were considered in this simulation. The data were acquired with $70 \times 70$ detector bins and 90 angular views over $360^{\circ}$ circular-orbit scan with $35 \mathrm{~mm}$ radius of rotation (ROR). As a comparison, the projection data were reconstructed using Feldkamp algorithm, 3D pinhole OS-EM algorithm with GPRC (4 iterations, 15 subsets) respectively. The image matrix was taken as $160 \times 160 \times 160$ with $(0.1875 \mathrm{~mm})^{3}$ voxel size. Figure $6(\mathrm{e})$ and $(\mathrm{g})$ show the comparison results between the two different reconstruction algorithms for the simple collimator blurring, which is the Feldkamp with Butterworth post-filtering (e) and the OS-EM reconstruction with GPRC but without any postfiltering $(\mathrm{g})$. We see that both algorithms could nearly resolve the $0.75 \mathrm{~mm}$ while using the $1.0 \mathrm{~mm}$ keel-edge pinhole collimator at the configuration of $35 \mathrm{~mm}$ ROR and 100 $\mathrm{mm}$ focal length. Figure 6(f) and (h) show the comparison results between the Feldkamp with post-filtering and the OS-EM reconstruction with GPRC but without any post-filtering for further projection blurred by the pinhole collimator response, Poisson distribution and statistical noise effects. As compared with the Feldkamp algorithm, 
the dedicated 3D OS-EM algorithm indicates an overall image quality improvement and noise reduction, and the system resolution is significantly recovered at relative real imaging condition.

A mathematical 3D Defrise phantom was further simulated to evaluate the performance of 3D OS-EM reconstruction algorithms for pinhole SPECT with circular and helical scan mode. The phantom consists of 8 uniform solid cylindrical disks, which can form 7 layers of hot activity plates. The diameter of the phantom is $27 \mathrm{~mm}$, the thickness of
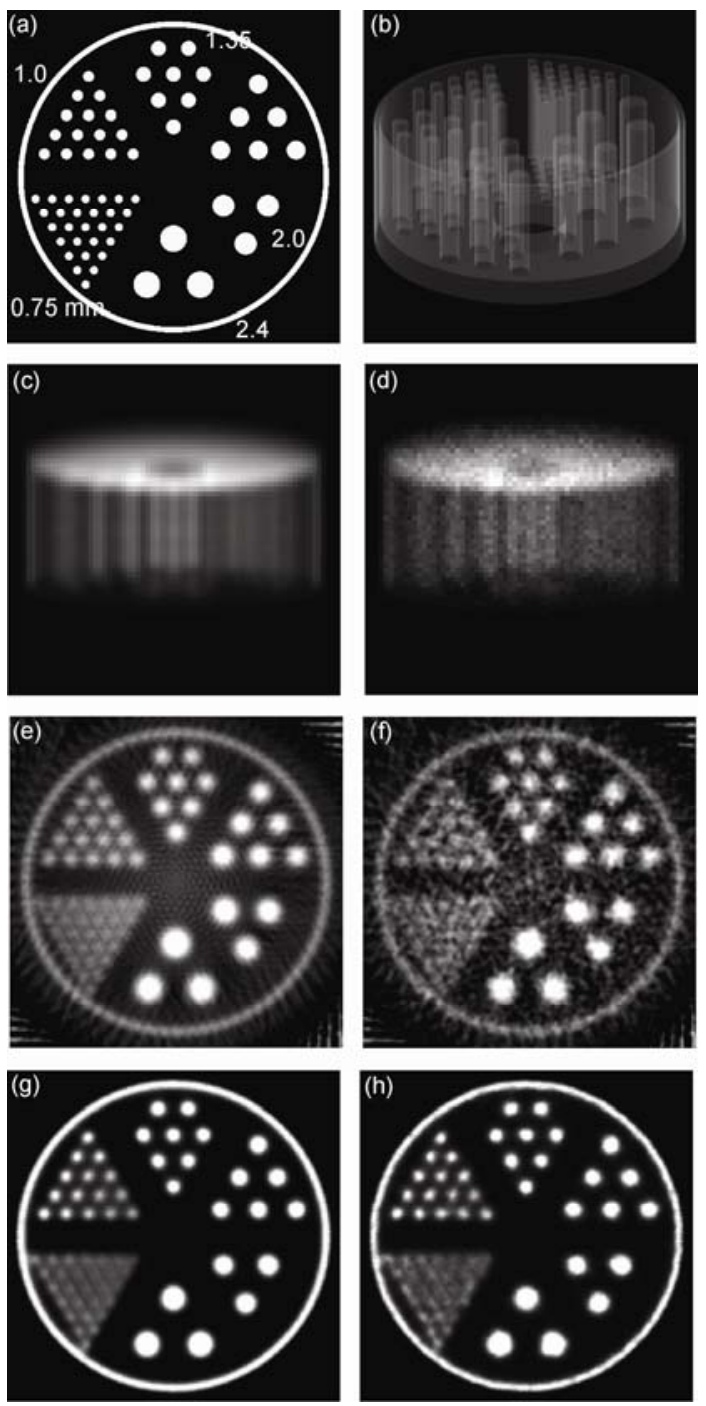

Figure 6 The computation simulation and image reconstruction of pinhole SPECT phantom imaging. (a) The trans-axial slice of the computerized 3D Jaszczak phantom; (b) 3D perspective rendering of the phantom; (c) the projection image of the computer simulation with keel-edge pinhole collimator response blurring; (d) the projection image of the computer simulation with keel-edge pinhole collimator response, Poisson distribution and statistical noise blurring; (e) and (g) are the comparison results of the trans-axial reconstruction images by the Feldkamp algorithm and the $3 \mathrm{D}$ pinhole OS-EM with compensation respectively for the simple collimator response blurring; (f) and (h) are the comparison results of the two reconstruction algorithms respectively for the collimator response, statistical fluctuations and noise blurring. each disk and the gap between the adjacent disks is 1.62 $\mathrm{mm}$. Figure 7 shows the trans-axial image (a), the central axial image (b), and the 3D perspective rendering(c) of the computerized phantom respectively. The noise-free projections were generated with $1.0 \mathrm{~mm}$ keel-edge pinhole collimator detector responses, and blurred with statistical fluctuation and noise contaminated, of which a sample projection is shown in Figure 7(d). The data were acquired with $70 \times 70$ detector bins with $37 \mathrm{~mm}$ radius of rotation (ROR). The projections were simulated through 90 angular views over $360^{\circ}$ by circular scan, and 180 angular views over $720^{\circ}$ by helical scan to provide sufficient sampling for covering the whole phantom in which the helical pitch was $0.15 \mathrm{~mm}$. Both scan modes acquired the data at the same angular interval $\left(4^{\circ}\right.$ by $360^{\circ} / 90$ and $\left.720^{\circ} / 180\right)$. The projection data were reconstructed respectively using the Feldkamp algorithm, and the 3D pinhole OS-EM algorithm with compensation by 4 iterations and 9 subsets. The image matrix was $100 \times 100 \times 100$ with $(0.3 \mathrm{~mm})^{3}$ voxel size. Figure $7(\mathrm{e})$, (f) show the central axial slice of reconstruction results by the Feldkamp and the circular 3D pinhole OS-EM algorithms respectively. Figure $7(\mathrm{~g})-(\mathrm{h})$ show the axial profiles of the reconstructed images in (e) and (f). The reconstruction with circular scan imaging can only resolve $\sim 1$ disk by Feldkamp and $\sim 3$ disks by 3D OS-EM at the configuration of $37 \mathrm{~mm}$ ROR and $100 \mathrm{~mm}$ focal length. Figure 7(i), (j) show the central axial reconstructed slice of the helical pinhole $3 \mathrm{D}$ OS-EM algorithm and its axial profile respectively. The results demonstrate that our developed algorithm can provide the expected reconstruction images of the phantom. However, the pinhole SPECT with the conventional circular scan induces severe axial artifacts on the reconstruction region far from the central orbit plane, while the helical pinhole SPECT can substantially reduce the axial blurring and provide almost non-artifact reconstructed image of the full phantom. According to the sufficient condition, the pinhole SPECT with circular-orbit scan provides insufficient projection data while imaging long objects, whereas helical-orbit scan could sample more sufficient projection to improve the axial spatial resolution of reconstruction image.

\subsection{Phantom imaging experiments}

To further evaluate the performance of the developed image reconstruction methods for our Micro-SPECT system, an experimental study was performed using the Ultra-Micro Hot Spot phantom (shown in Figure 8(a), Data Spectrum, Hillsborough, NC, USA) filled with $\sim 5 \mathrm{mCi}{ }^{99 \mathrm{~m}} \mathrm{Tc}$ pertechnetate in water solution. The detailed specification about the phantom is identical to the computerized 3D Jaszczak phantom which was simulated in section 2.1. The experiment was performed with $0.7 \mathrm{~mm}$ keel-edge pinhole collimator. Each frame of the available projection was taken as $70 \times 70$ detector bins. The radius-of-rotation (ROR) was $\sim 35$ $\mathrm{mm}$, which results in a magnification of $\sim 3$. The projection 

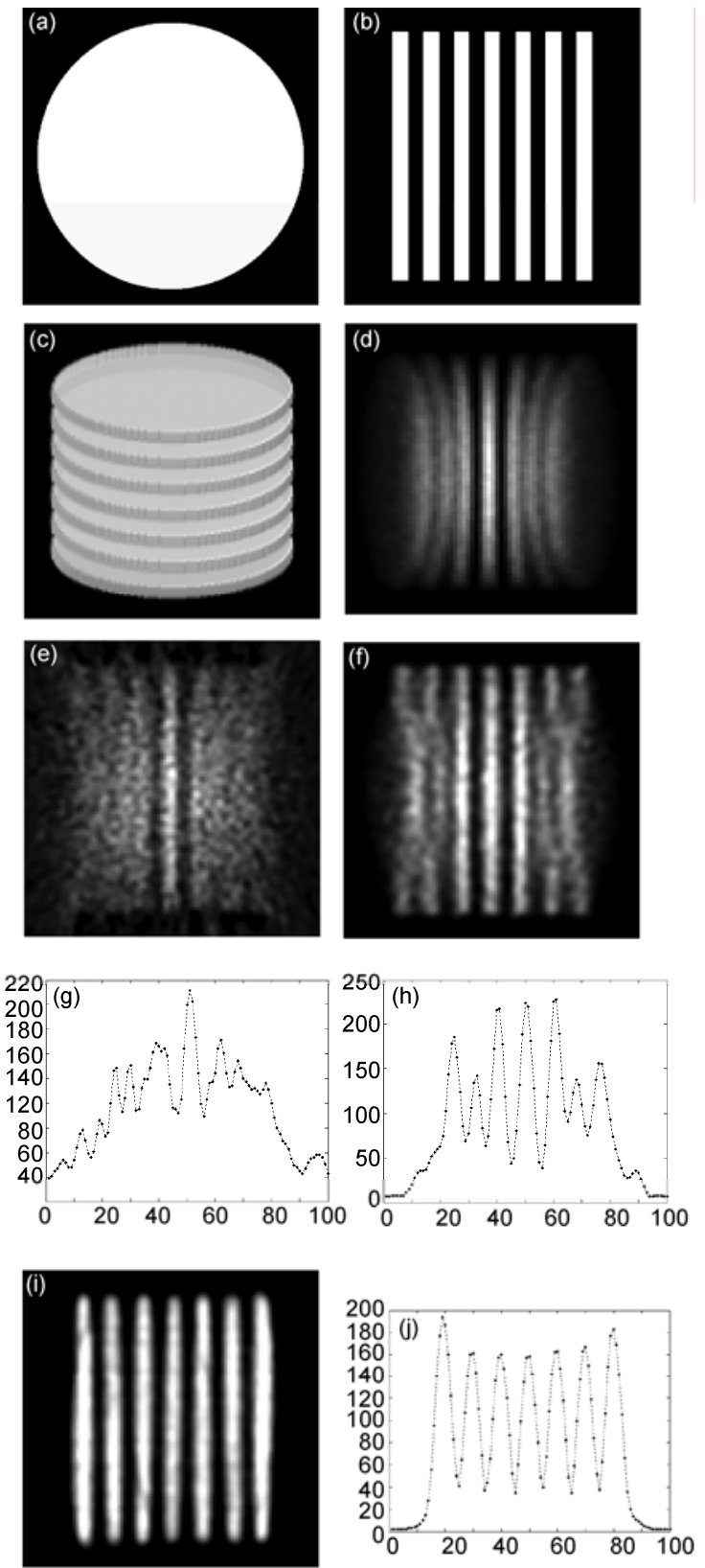

Figure 7 The computation simulation and image reconstruction comparison results between circular and helical single pinhole SPECT. (a) The trans-axial slice of the computerized 3D Defrise phantom; (b) the central axial slice of the phantom; (c) 3D perspective rendering of the phantom; (d) a sample projection image of the computer simulation; (e) the central axial reconstruction image of the Feldkamp algorithm; (f) the central axial reconstruction image of the 3D pinhole OS-EM with circular scan; $(\mathrm{g})$ the axial profile of the slice in (e); (h) the profile of the slice in (f); (i) the central axial reconstruction image of the 3D pinhole OS-EM with helical scan; (j) the profile of the slice in (i).

data were acquired by 90 equal angular views over $360^{\circ}$ with circular scan, and the acquisition time was $30 \mathrm{~s}$ per view. Figure 8(b) shows a sample projection image by our Micro-SPECT system. The image was reconstructed as $160 \times 160 \times 160$ matrix with $(0.1875 \mathrm{~mm})^{3}$ voxel size. As a comparison, we also performed the image reconstruction using the Feldkamp algorithm, and the 3D pinhole OS-EM algorithm with collimator-detector response compensation (4 iterations, 15 subsets) respectively. Figure 8(c), (d) shows the comparison results of the reconstructed transaxial slice between the two algorithms, which is the Feldkamp reconstruction with Butterworth post-filtering (c) and the OS-EM reconstruction with GPRC but without any post-filtering (d). As compared with the Feldkamp algorithm, the dedicated 3D OS-EM algorithm indicates an overall image quality improvement with effective degrading compensation, and the system resolution is significantly improved to well recover the $0.75 \mathrm{~mm}$ rods with the $0.7 \mathrm{~mm}$ keel-edge pinhole collimator at the configuration of $\sim 35 \mathrm{~mm}$ ROR and $100 \mathrm{~mm}$ focal length by our Micro-SPECT system.

We also performed phantom experiment using an UltraMicro Defrise phantom (shown in Figure 9(a), Data Spectrum, Hillsborough, NC, USA) to further validate our pinhole SPECT imaging system. The performance of the image reconstruction we developed was also evaluated by this phantom experiment. The phantom consisted of a cylindrical volume (both the inside diameter and the height are of $2.8 \mathrm{~cm})$. The insert of the cylinder consists of 8 solid disks (2.7 $\mathrm{cm}$ diameter) connected by solid screws. The thickness of each disk and the gap between them are $1.62 \mathrm{~mm}$ respectively. The phantom was filled with $\sim 5 \mathrm{mCi}$ of ${ }^{99 \mathrm{~m}} \mathrm{Tc}-$ pertechnetate in water solution. The SPECT imaging with both circular and helical scans were performed at the radius-of-rotation (ROR) of $\sim 37 \mathrm{~mm}$ with $1.0 \mathrm{~mm}$ keel-edge pinhole aperture. The projection data were acquired with 90 angular views over $360^{\circ}$ by circular scan, and 180 angular views over $720^{\circ}$ by helical scan in which the helical pitch was $0.15 \mathrm{~mm}$ to cover the whole phantom from the bottom
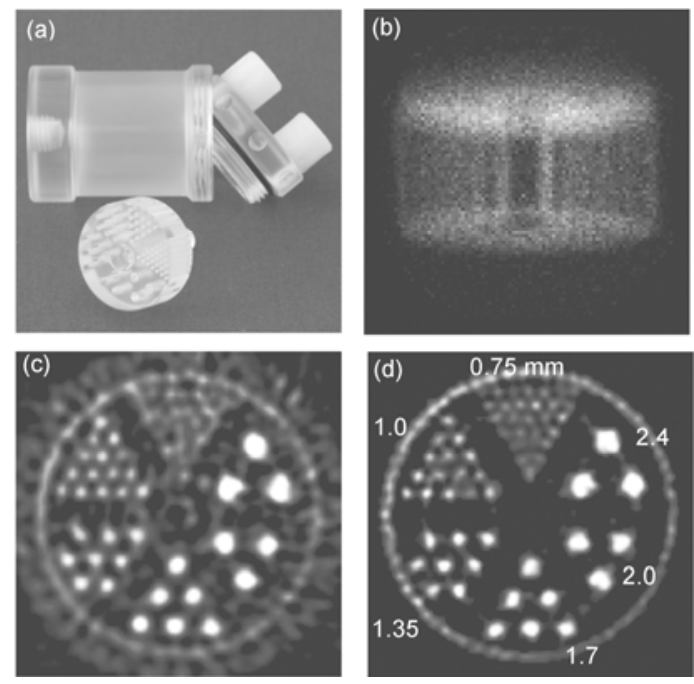

Figure 8 The pinhole SPECT imaging experiments using Ultra-Micro Hot-Spot phantom (Data Spectrum, Hillsborough, NC, USA). (a) A photograph of the phantom; (b) one sample projection image by our Micro-SPECT; (c) the reconstructed trans-axial image using Feldkamp algorithm w/ post-filtering; (d) The reconstructed trans-axial image using 3D pinhole OS-EM w/ GPRC. 

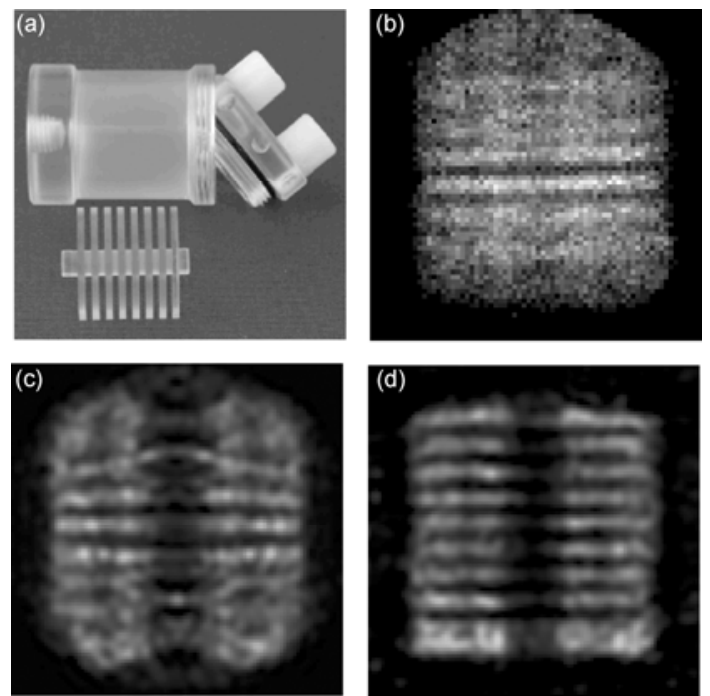

Figure 9 The pinhole SPECT imaging experiments using Ultra-Micro Defrise phantom (Data Spectrum, Hillsborough, NC, USA). (a) A photograph of the phantom; (b) one sample projection image by our Micro-SPECT; (c) the reconstructed axial central image using 3D pinhole OS-EM with circular scan; (d) the reconstructed axial central image using 3D pinhole OS-EM with helical scan.

to the top over all the projections. The acquisition time was 30 seconds per view. Figure 9(b) shows one sample projection of the phantom SPECT imaging. The size of the available detector bins was taken as $70 \times 70$. The two sets of projection data were reconstructed using the dedicated circular and helical 3D pinhole OS-EM algorithm with compensation by 4 iterations, 9 subsets and the image matrix was $100 \times 100 \times 100$ with $(0.3 \mathrm{~mm})^{3}$ voxel size. The results of the reconstructed central-axial images of the phantom from both the circular and helical SPECT imaging were shown in Figure 9(c) and (d). With the same ROR and pinhole aperture, the helical pinhole SPECT scan can significantly improve the axial FOV to reconstruct all the 8 disks of the phantom while the circular pinhole SPECT scan can only reconstruct $\sim 3$ disks due to a limited FOV of pinhole geometry and insufficient projection sampling. For the circular scan, the non artificial reconstruction is only achieved in the limited field of central plane of the circular orbit, and the regions far from the central plane induce substantial artifacts.

\subsection{Small animal imaging experiments}

To validate the proposed fully $3 \mathrm{D}$ imaging methods by our Micro-SPECT system for the pre-clinical small animal studies, a mouse bone metabolism imaging experiment with a helical scan was performed for a further evaluation using a $0.7 \mathrm{~mm}$ keel-edge pinhole aperture. A 20-gram normal mouse with the body length less than $100 \mathrm{~mm}$ was injected with $\sim 2 \mathrm{mCi}{ }^{99 \mathrm{~m}} \mathrm{Tc}-\mathrm{MDP}$ through its caudal vein. The radius-of-rotation of the helical SPECT scan was $\sim 39 \mathrm{~mm}$. To obtain the whole-body image of the mouse, a total of 180 angular views over $720^{\circ}$ and the helical pitch of $0.5 \mathrm{~mm}$ were performed in the helical scan. The acquisition time was 30 seconds per view. The size of the available detector bins was taken as $70 \times 70$ and the reconstructed image matrix was $180 \times 180 \times 360$ with $(0.3 \mathrm{~mm})^{3}$ voxel size to cover the whole mouse. The images were reconstructed using our dedicated voxel-driven 3D pinhole OS-EM algorithm with compensation for 9 subsets with 5 iterations. We also implemented the maximum intensity projection algorithm (MIP) to present an effective 3D volume rendering for image reconstruction of emission computed tomography. Figure 10(a) shows some projection images of the mouse bone scan by our helical pinhole SPECT. Figure 10(b) shows the reconstruction images with the posterior (left) and the lateral (right) displays of MIP, in which the bladder was removed. In spite of the relative lower counts in the projection data and much smaller diameter of the available detector (98 $\mathrm{mm}$ by $70 \times 1.4 \mathrm{~mm}$ ) than the commercial clinical gamma camera, the reconstruction with our helical pinhole OS-EM algorithm can still provide fair good images. High spatial resolution is achieved in the bone scan imaging, where small bone structure such as the spine and ribs are well resolved. The results demonstrate that the helical SPECT is reliable and alternative to provide high resolution and decent whole-body imaging for long small animal studies.

\section{Discussion and conclusions}

In this study, we have successfully developed full 3D imaging reconstruction for pinhole SPECT imaging with different scan modes. The performance of the reconstruction algorithms and quantitative compensation method were evaluated using computer simulations, phantom imaging experiments, and small animal imaging experiment studies based on our Micro-SPECT imaging system. The results from the simulations and experiments of phantom imaging show that the developed 3D pinhole OS-EM reconstruction algorithm with collimator-detector response compensation provides fairly good reconstruction images and recover a real high resolution for both circular and helical scans. The helical pinhole SPECT can significantly improve the axial FOV and axial resolution as compared with the circular scan mode. The mouse bone imaging experiment demonstrates that the decent whole-body small animal imaging with high resolution is achievable with the helical SPECT, which is an effective method for long object imaging.

However, the quantitative evaluation of the image reconstruction method in this paper was very limited because of the relative low counts of the projection data in the imaging experiments. The study on the trade-off between resolution and noise in the reconstructed images needs further computer simulations and experiments to perform and obtain sufficient quantitative results. 

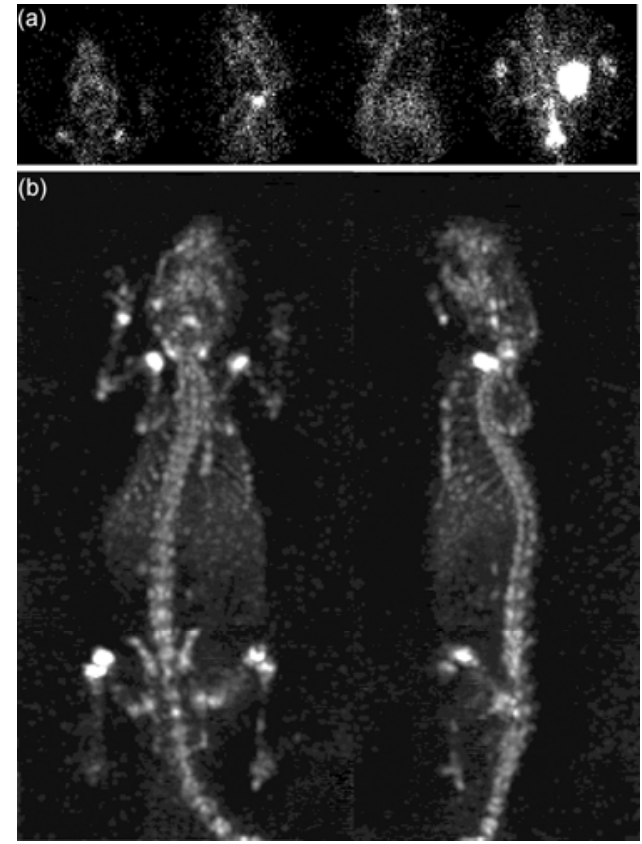

Figure 10 The pinhole SPECT imaging of mouse bone injected with ${ }^{99} \mathrm{~m}$ Tc-MDP. (a) Four selected sample projections of the mouse's head, neck, chest and pelvis during the helical scan; (b) the reconstruction images displayed by the posterior (left) and the lateral (right) 3D volume renderings with MIP.

In conclusion, we have successfully developed a set of valid fully $3 \mathrm{D}$ image reconstruction techniques for single-pinhole SPECT imaging. These techniques can be easily extended to multi-pinhole SPECT imaging.

This work was supported in part by the National Natural Science Foundation of China (30570520 \& 10875162).

1 Blankenberg F G. Molecular imaging with single photon emission computed tomography. IEEE Eng Med Biol, 2004, 23: 51-57

2 Meikle S R, Kench P, Kassiou M, et al. Small animal SPECT and its place in the matrix of molecular imaging technologies. Phys Med Biol, 2005, 50: R45-R61

3 Kupinski M A, Barrett H H. Small-animal SPECT Imaging. New York: Springer Press, 2005

4 Qi Y J. High-resolution SPECT for small-animal imaging. Nucl Sci Tech, 2006, 17: 164-169

5 Tsui B M W, Wang Y, Yoder B C, et al. Micro-SPECT. In: Proceedings of IEEE International Symposium on Biomedical Imaging, 2002, IEEE Press, 373-376

6 Furenlid L R, Wilson D W, Chen Y, et al. FastSPECT II: A second-generation high-resolution dynamic SPECT imager. IEEE Trans Nucl Sci, 2004, 51: 631-635

7 Beekman F, Have F, The pinhole: Gateway to ultra-high-resolution three-dimensional radionuclide imaging. Eur J Nucl Med Mol Imaging, 2007, 34: 151-161

8 Qi Y J. Optimized collimator designs for small animal SPECT imaging with a compact gamma camera. In: 27th Annual International Conference of the IEEE Engineering in Medicine and Biology Soci- ety, 2005, IEEE Press, 1780-1782

9 Metzler S D, Bowsher J E, Jaszczak R J. Geometrical similarities of the Orlov and Tuy sampling criteria and a numerical algorithm for assessing sampling completeness. IEEE Trans Nucl Sci, 2003, 50: $1550-1555$

10 Metzler S D, Greer K L, Jaszczak R J. Helical pinhole SPECT for small-animal imaging: A method for addressing sampling completeness. IEEE Trans Nucl Sci, 2003, 50: 1575-1583

11 Tuy H K. An inversion formula for cone-beam reconstruction. SIAM J Appl Math, 1983, 43: 546-552

12 Smith B D. Image reconstruction from cone-beam projections: Necessary and sufficient condition and reconstruction methods. IEEE Trans Med Imaging, 1985, 4: 14-25

13 Orlov S S. Theory of three dimensional reconstruction. I. condition for a complete set of projections. Sov Phys Crystallogr, 1975, 20 312-314

14 Cherry S R, Sorenson J, Phelps M. Physics in Nuclear Medicine. 3rd ed. Philadelphia, Pa: Saunders/Elsevier Science Press, 2003

15 Barrett H H, Mayer K J. Foundations of Image Science. New York Wiley-Interscience Press, 2003

16 Herman G T. Fundamentals of Computerized Tomography: Image Reconstruction from Projections. San Francisco: Academic Press, 1980

17 Natter F, Wubbeling F. Mathematical Methods in Imaging Reconstruction. SIAM Press, 2001

18 Shepp L A, Vardi Y. Maximum likelihood reconstruction for emission tomography. IEEE Trans Med Imaging, 1982, 1: 113-122

19 Lange $\mathrm{K}$ and Carson R. EM reconstruction algorithms for emission and transmission tomography. J Comput Assist Tomogr, 1984, 8: 306-316

20 Qi J Y, Leahy R M. Iterative reconstruction techniques in emission computed tomography. Phys Med Biol, 2006, 51: R541-R578

21 Hudson H M, Larkin R S. Accelerated image reconstruction using ordered subsets of projection data. IEEE Trans Med Imaging, 1994, 13: 601-609

22 Zhang X Z, Dai Q S, Qi Y J. Implementing analytical geometric and penetration response correction for keel-edge pinhole SPECT image reconstruction. In: IEEE Nuclear Science Symposium Conference Record, 2008, IEEE Press, 5255-5259

23 Zhang X Z, Chen F F, Qi Y J, et al. Unified Geometric Calibration and Image Registration for Detached Small Animal SPECT/CT. In: IEEE Nuclear Science Symposium Conference Record, 2009, IEEE Press, 3318-3321

24 Beque D, Nuyts J, Bormans G, et al. Characterization of pinhole SPECT acquisition geometry. IEEE Trans Med Imaging, 2003. 22: 599-612

25 Wang Y. Development and application of high-sensitivity and high-resolution fully 3D SPECT imaging techniques using two different collimator designs. Doctor Dissertation. Chapel Hill: The University of North Carolina, 2004

26 Wang Y, Tsui B M W. Pinhole SPECT with different data acquisition geometries: usefulness of unified projection operators in homogeneous coordinates. IEEE Trans Med Imaging, 2007, 26: 298-308

27 Press W H, Teukolsky S A, Vetterling W T, et al. Numerical Recipes in C: The Art of Scientific Computing, 2nd ed. Cambridge, U.K.: Cambridge University Press, 1992

28 Zhang M J, Qi Y J. Readout electronics of a high-resolution gamma camera (in Chinese). Nuclear Tech, 2007, 30: 629-632

29 Qi Y J, Zhang M J, Zhao C L, et al. Performance comparison of subtractive resistive readout with conventional resistive readout for high-resolution compact gamma camera. In: IEEE Nuclear Science Symposium Conference Record, 2007, IEEE Press, 3725-3728

30 Zhang M J. Study of readout electronics of Micro-SPECT (in Chinese). Master Thesis. Beijing: Graduate University of Chinese Academy of Sciences, 2007

Open Access This article is distributed under the terms of the Creative Commons Attribution License which permits any use, distribution, and reproduction in any medium, provided the original author(s) and source are credited. 